\title{
Editorials
}

\section{The hidden cost of anesthesia}

\author{
David Bevan MB MRCP FRCA FRCPC
}

A T first glance, the examination by Pradervand et al. in this issue of the Canadian Journal of Anesthesia, ${ }^{1}$ of anesthesia costs for patients undergoing laparoscopic cholecystectomy provides no new information: anesthesia accounts for approximately one third of their total hospital costs. Macario et al. reported very similar findings in 1994:2 have we learned nothing in the last decade? Closer inspection reveals some interesting differences between the two reports. First, comparing North American with European practice: hospital stay for the same operation was four days in Lausanne compared with one day in Stanford. Second, Pradervand et al. calculated all anesthesia costs including compensation of personnel, physicians, nurses and technicians which was ignored by Macario et al. Finally, the authors suggest that any real attempt at decreasing operative costs should focus on the areas of greatest expense particularly in the operating room (OR; excluding anesthesia), which is responsible for $37 \%$ of the total hospital cost and the ward $-31 \%$. Cost reduction in the recovery room is of marginal economic value. Each of these issues merits further discussion.

During the last ten to 15 years, to save hospital costs, huge efforts have been made in North America to minimize the length of hospital stay. Most surgical patients are admitted on the day of operation and discharged "home" as soon as (or before) they can manage independent life! The logic is irrefutable - hospital beds are very expensive to staff and maintain. Shifting these "hotel" costs reduces hospital expense although we often forget to whom the cost is shifted and what additional expenditures are incurred with their downstream effects. Much of the cost is shifted from hospital to the patient and his/her family. Driving from North Bay to Toronto in early February to reach University Avenue to arrive in the same day admission unit by 6 a.m. before major surgery is no fun. The family takes time off work, pays to stay in the nearest hotel for a couple of days and repeats the experience on discharge, at some uncertain time in the future. Additional services need to be provided, both locally and in Toronto, for pre-admission testing and evaluation, integration of special tests such as echocardiography mean inefficient services if they are to be always available. Most hospitals allocate one or more anesthesiologists to pre-admission and/or consult clinics to perform assessments that were previously performed on the evening before surgery.

It is not surprising that we have failed to train sufficient anesthesiologists to perform all the necessary tasks. Current estimates are that, in Ontario, we are short by about 100, of a present total of 850. ${ }^{\mathrm{A}}$ However, hospital costs have been reduced. At least, beds have been closed and nurses released but to leave only the sickest patients in hospital and a huge wave of antagonism and suspicion by nursing professionals who feel they were made the scapegoats for health care spending. We should not be surprised at our current nursing shortages and the pay settlements necessary to attract more nurses into a system that so recently rejected them!

It is unfortunate that it is not possible to compare the actual cost of laparoscopic cholecystectomy between Lausanne and Stanford because the data are presented as percentages of total costs and not in dollars/francs. Although the average stay in Lausanne was 4.3 days, one patient remained in hospital for 22 days and the cost of hospital stay represented $56 \%$ of the total. In Baltimore, Fleisher et al. reported that the cost of laparoscopic cholecystectomy was decreased in ambulatory $v s$ inpatients (from $\$ 4,000$ to $\$ 2,000$ ). ${ }^{3}$ However, that study did not include health professional compensation and

A Ryten E. A physician work force planning model 2001. The report, prepared for the Association of Canadian University Departments of Anesthesia may be consulted at www.anesthesia.org/acuda.

From the Department of Anesthesia, University Health Network, Toronto General Hospital, Toronto, Ontario, Canada. Address correspondence to: Dr. David Bevan, Department of Anesthesia, University Health Network, Toronto General Hospital, 585 University Avenue, Bell Wing 4-644, Toronto, Ontario M5G 2C4, Canada. Phone: 416-340-5164; Fax: 416-340-3698;

E-mail: david.bevan@uhn.on.ca 
there was a surprising difference in OR cost between inpatients and ambulatory patients $(\$ 1,800$ vs $\$ 760)$. What is the cost of a few days in the nearest hotel?!

North American reporters have been reluctant to include the cost of compensation to professionals in their economic models although Moote in London, Ontario, showed that over $90 \%$ of the cost of providing a postoperative patient controlled analgesia service was to remunerate the physicians supplying the service. ${ }^{4}$ Health care is expensive and the most qualified practitioners are the most expensive component of the system. Similar comparisons can be made with other professions (e.g., judges, lawyers, court costs) and industries (air-line pilots, attendants, planes). However, it is difficult to argue with Moote that "in the race to reduce cost, we must strive to protect essential services for patients who need them most." The provision of anesthesia services requires individuals of different training and experience. The concept of an "anesthetic team" is attractive to make use of a mix of individuals to provide the safest anesthetic environment for every patient but there is no justification to replace physician anesthesiologists with less qualified inadequately supervised personnel.

Patients should expect prompt, safe surgical care at an economic cost. Such a service may not be the "cheapest" in terms of absolute dollars. Drugs and techniques, such as analgesia and anti-nausea therapy, should be included because they improve quality of the surgical experience. We should not confuse cost (the lowest price for each procedure) with efficiency (the most economic price for the largest number of patients). The present study gives us two messages:

1) Cost reductions should focus on the most expensive areas - ward and OR.

2) Salaries and wages are the most expensive component of health care expenditure.

Perhaps, we should base our care on making the maximum use of our most scarce resources - anesthesiologists and nurses. The best care to the largest number of patients will be achieved if we provide adequate numbers of appropriately trained personnel and ensure that the environment supports them to provide maximal utilization. The future of ambulatory care is not yet clear. It is attractive to believe that we should be providing health care like convenience stores - $24 \mathrm{hr}$ a day, seven days a week. Such an idea is predicated on the false premise that buildings are the most expensive component of the system. This year, in most Canadian hospitals, anesthesiology is the scarce resource!

\section{Le coût caché de l'anesthésie}

À première vue, l'examen réalisé par Pradervand et coll., dans le présent numéro du Journal ${ }^{1}$ sur les coûts d'une cholécystectomie laparoscopique, n'apporte pas d'informations nouvelles : l'anesthésie constitue environ le tiers des coûts hospitaliers totaux. Macario et coll. ont fait des constatations très similaires en $1994^{2}$ : n'avonsnous rien appris pendant la dernière décennie ? Une lecture plus attentive révèle d'intéressantes différences entre les deux articles. D'abord, la comparaison de la pratique en Amérique du Nord et en Europe montre que pour une même opération, le séjour hospitalier est de quatre jours à Lausanne et de un à Stanford. Ensuite, Pradervand et coll. ont calculé tous les coûts de l'anesthésie, incluant la rémunération du personnel, des médecins, des infirmières et des techniciens dont Macario et coll n'ont pas tenu compte. Enfin, les auteurs concluent que toute intervention visant à diminuer les coûts opératoires devra se centrer sur ce qui génère les dépenses les plus importantes, particulièrement la salle d'opération ( $\mathrm{SO}$, à l'exclusion de l'anesthésie) qui est responsable de $37 \%$ du coût total d'hospitalisation et l'unité de soins - $31 \%$. La réduction du coût de la salle de réveil n'a qu'une valeur économique marginale. Chacun de ces aspects mérite plus ample réflexion.

Pendant les dix à quinze dernières années, pour économiser dans les hôpitaux d'Amérique du Nord, de grands efforts ont porté sur la réduction du séjour hospitalier. La plupart des opérés étaient pris en charge le jour de l'opération et retournaient "à la maison" aussitôt qu'ils recouvraient leur autonomie ou même avant ! La logique est irréfutable - il en coûte très cher de pourvoir en personnel et de maintenir l'hospitalisation. Le déplacement de ces coûts "hôleliers" réduit les dépenses de l'hôpital, mais on oublie trop souvent à qui ils reviennent et quelles dépenses supplémentaires sont encourues avec leurs effets en aval. La plus grande partie du coût va ainsi de l'hôpital vers le patient et sa famille. Rouler entre North Bay et Toronto au début de février pour atteindre University Avenue et arriver à l'unité à six heures le jour de l'admission avant une intervention chirurgicale importante, n'est pas très drôle. La famille s'absente du travail, paye l'hôtel le plus près pendant quelques jours et refait la même chose quand le patient quitte l'hôpital à un moment indéterminé, un peu plus tard. Des services complémentaires doivent être offerts, localement et à Toronto, car les tests et l'é- 
valuation préalables à l'admission, l'intégration de tests spéciaux comme l'échocardiographie se révèlent des services inefficaces s'il faut les maintenir constamment disponibles. La plupart des hôpitaux affectent au moins un anesthésiologiste aux cliniques de pré-admission et/ou de consultation, ce qui leur permet de procéder à l'évaluation faite auparavant la veille de l'opération.

Il n'est pas surprenant que nous ayons échoué à former suffisamment d'anesthésiologistes pour toutes les tâches à réaliser. Les estimations actuelles font état d'une pénurie d'une centaine de spécialistes en Ontario sur un total de $850 .^{\mathrm{A}}$ Les coûts hospitaliers ont toutefois été réduits. On a au moins diminué le nombre de lits disponibles et d'infirmières, mais pour ne laisser que les malades les plus sévères à l'hôpital et une gigantesque vague de protestation et de méfiance chez le personnel infirmier qui se voit comme le bouc émissaire des dépenses de santé. Il ne faut pas s'étonner de la pénurie d'infirmières et de payer ce qu'il faut pour en attirer davantage dans un système qui les a rejetées il n'y a pas si longtemps!

Il est regrettable de ne pouvoir comparer le coût réel de la cholécystectomie laparoscopique réalisée à Lausanne ou à Stanford, car les données sont présentées en pourcentage des coûts totaux et non en dollars ou en francs. Quoique le séjour moyen était de 4,3 jours à Lausanne, un patient est demeuré à l'hôpital pendant 22 jours et le coût du séjour représentait $56 \%$ du total. Fleisher et coll. ont constaté qu'à Baltimore le coût de la cholécystectomie laparoscopique était plus bas pour un patient ambulatoire vs un patient hospitalisé (passant de $4000 \$$ à $2000 \$) .{ }^{3}$ Cependant, cette étude ne comprend pas le salaire des professionnels de la santé et on note une différence surprenante du coût de la SO entre les patients hospitalisés et ambulatoires ( $1800 \$ v$ v $760 \$$ ). Quel est le coût de quelques jours à l'hôtel le plus proche ?!

Les rédacteurs de rapport nord-américains hésitent à inclure le coût du salaire des professionnels dans leurs modèles économiques, bien que Moote, de London en Ontario, ait montré que plus de $90 \%$ du coût d'un service d'analgésie postopératoire autocontrôlée sont liés à la rémunération des médecins. ${ }^{4}$ Les soins de santé sont dispendieux et les médecins les plus qualifiés représentent la composante la plus chère du système. Des comparaisons similaires peuvent être faites avec d'autres professions (les juges, les avocats, les frais judiciaires) et d'autres industries (pilotes de ligne, hôtesses de l'air, avions). Il est toutefois difficile de contester Moote sur le fait que "dans la course à la réduction des coûts, nous devons tenter de protéger les services essentiels pour les patients qui en ont le plus besoin." La prestation des services d'anesthésie exige des individus de formation et d'expérience dif- férentes. Le concept d'une "équipe anesthésique" est attrayant, qui emploierait différentes personnes pour assurer un environnement anesthésique des plus sûrs pour chaque patient, mais rien de peut justifier de remplacer les anesthésiologistes par du personnel moins qualifié et insuffisamment supervisé.

Les patients doivent compter sur des soins chirurgicaux rapides et sûrs à coût économique. Ce service ne sera peut-être pas le "moins cher" en termes de dollars absolus. Les médicaments et les techniques, comme l'analgésie et le traitement antiémétique, devraient être inclus parce qu'ils améliorent la qualité de l'expérience chirurgicale. Nous ne devons pas confondre le coût (le meilleur prix pour chaque intervention) et l'efficacité (le prix le plus économique pour le plus grand nombre de patients). La présente étude contient deux messages :

1) Les réductions de coût doivent être centrées sur les composantes les plus dispendieuses - les unités de soins et la SO.

2) Les salaires et les rémunérations sont la partie la plus chère des dépenses de santé.

Nos soins devraient peut-être reposer sur l'utilisation de nos ressources les plus rares - les anesthésiologistes et le personnel infirmier. On pourra offrir les meilleurs soins au plus grand nombre de patients si on peut trouver un nombre suffisant de personnes bien formées et s'assurer que l'environnement leur permet une utilisation maximale. L'avenir des soins ambulatoires n'est pas encore clair. Il est tentant de croire qu'on pourrait donner des soins de santé sur le modèle des dépanneurs - tous les jours, 24 h sur 24. Cette idée est fondée sur la fausse prémisse que les immeubles sont la composante la plus dispendieuse du système. Pourtant, cette année, dans la plupart des hôpitaux canadiens, l'anesthésiologie est la ressource rare!

\section{References}

1 Pradervand Mooser M, Gardaz J-P, Capt H, Spabn DR. Le coût relatif de l'anesthésie pour la cholécystectomie laparoscopique est peu élevé. Can J Anesth 2002; 49; 540-44.

2 Macario A, Vitez TS, Dunn B, McDonald T. Where are the costs in perioperative care? Analysis of hospital costs and changes for inpatient surgical care. Anesthesiology 1995; 83: 1138-44.

3 Fleisher LA, Yee K, Lillemoe KD, et al. Is outpatient laparoscopic cholecystectomy safe and cost-effective? A model to study transition of care. Anesthesiology 1999; 90: 1746-55.

4 Moote CA. Postoperative pain management - back to basics. Can J Anaesth 1995; 42: 453-7. 